\section{CASE OF CONGENITAL MALFORMATION OF THE HEART.}

BY GEORGE W. BALFOUR, M.D., PHYGICIAN TO THE ROYAL INFIRMARY, EDINBURGH.

(Case reported by Mr. KELLY, Clinical Clerk.)

ANNE C-, aged nineteen, a dressmaker, born in Aberdeen, and residing at Leith, was admitted to bed 10, ward xiii., on December 1st, 1873, complaining of great pain over the præcordia, extending to the left back and shooting down the left arm, and of a bad sore-throat. The patient is usually deeply cyanotic, but varies in complexion; the digits on all four extremities are clubbed in a very marked degree, and the nails aduncated.

Her mother stated that last February (1873), after receiving a fright, her daughter was seized with a sudden pain in the left side, which finally settled over the præcordia. At that time she suffered much from palpitation and general feeling of oppression in the chest. This state of matters lasted till May. After a short period of comparative health she was, in June, seized with a shivering fit, and shortly after coughed up two mouthfuls of dark blood and several more of a lighter colour. Since then she has always been ailing. Her mother also stated that ever since birth she had been of a blue colour, specially marked after any exertion and in cold weather. Shortly after her birth her father contracted syphilis, which he communicated to her mother, and, through her mother, to herself and to all the children born subsequently. So that, while ber brothers and sisters born previously are healthy, she and her mother both suffer from acquired syphilis, and those born subsequently from congenital syphilis.

Anne $\mathrm{C}-$ - is fairly developed, rather above the average height, and weighs 8 st. $3 \mathrm{lb}$. Her facial expression is dull; her complexion is of a rosy hue, sometimes livid; her lips generally, and her clubbed finger-tips always, livid, and the same remark may be made of her ankles and toes; her skin generally is not discoloured, is sometimes warm, moist, and clammy, at others cold and dry ; temperature $98.4^{\circ}$. Her pulse varies from 68 to 75 per minute, is feeble, and equal at both wrists. The jugular veins are small, but distinct pulsation is visible in them. She has dyspnoea on the slightest exertion, no palpitation, but severe pain over the præcordia. On inspection the chest appears normal; no pulsation anywhere visible. On palpation no impulse is anywhere perceptible, even when she leans forward, but a considerable thrill is to be felt over the base of the heart. On percussion from above downwards, one inch from the left of the sternum, cardiac dulness begins at the level of the upper edge of the third rib, and extends down to the liver dulness. At the level of the fourth rib dulness begins three-quarters of an inch to the right of the sternum, and extends to the left for a distance of four inches and threequarters. The aortic dulness is found to extend upwards to within one inch of the top of the sternum. On auscultation a loud systolic murmur is distinctly audible over the whole cardiac area, propagated with more distinctness towards the left than towards the right axilla. By careful auscultation posteriorly a faint systolic murmur is audible, evidently due to propagation through the bones. In the mitral area (between the fifth and sixth ribs, two inches and a half to the left of the sternum) this systolic murmur is shrill and distinct, followed by a faint second sound. In the tricuspid area (sternal end of the fourth, fifth, and sixth ribs on the left side) the systolic murmur is louder, more prolonged, and followed by a more distinct second sound. In the aortic area (sternal end of the second rib on the right side) the systolic murmur is shrill, distinct, and followed by a distinct second sound, of ten markedly accentuated, both sounds being propagated into the carotids. In the pulmonary area (sternal end of the third rib on the left side) the systolic murmur is louder and rougher than in any other part of the cardiac area, and followed by a second sound which, though always distinct, is at times quite accentuated; when it is most distinct the aortic accentuation seems to be least marked. Her respiration is 20 per minute; dyspnoea on the slightest exertion; pain in the chest; no cough or expectoration; voice hoarse; pulmonary physical signs normal. With the exception of sleeplessness, occasional shooting pain over the left temple, and the præcordial pain already referred to, her nervous system is normal. There is considerable difficulty of swallowing, from syphilitic ulceration of the uvula and swollen and congested tonsils, and slight atonic dyspepsia, digestive system otherwise normal. The urine is of a pale-sherry colour, acid, sp. gr. 1020, containing a small amount of albumen; otherwise normal; no deposit. Menstruation regular.

Remarks.-This history, thus concisely narrated, contains within itself all the elements for an accurate diagnosis. The history and symptoms of acquired syphilis, though sufficiently distressing in themselves, are purely episodic in relation to the more serious affection under which this patient labours-an affection which, from its congenital character, has necessarily embittered all her life and must shorten her days. The cyanotic condition of her surface, which has prevailed from birth, and the marked clubbing of all her digital extremities, point with unfailing accuracy to the existence of some congenital central lesion of her circulation. With regard to the production of cyanosis, three views are prevalent: first, there is the theory that it depends upon intermixture of the arterial and venous blood currents; second, that it is caused by venous congestion, depending upon obstruction to the onward flow of the blood current, from whatever cause; and, third, that it arises from a combination of the two preceding causes. Clinical experience, however, teaches us that even where the obstruction is very great, as in extreme constriction of the mitral opening-such as one now before me, which, though somewhat enlarged by long maceration in spirit, only measures five millimetres by eight,-the cyanosis is never so great as in those cases where, from congenital defect, intermixture of the two kinds of blood is possible. Whatever, therefore, may be the explanationand it is a subject still open to inquiry,-there can be no doubt that, though the absence of great cyanosis does not disprove the structural possibility of intermixture of the blood currents, its presence is a certain proof of its occurrence, and therefore of the presence of those malformations which render it possible. This is confirmed in the present case by the existence of marked clubbing of the digital extremities-a condition which must have a cardiac origin if it extend, as in this case, to all four extremities in any marked degree. The presence of these conditions therefore enables us to conclude with certainty that in this case we have to do with congenital cardiac malformation, involving some condition capable of permitting intermixture of the arterial and venous blood currents. Now, apart from congenital deficiencies of the septa alone, which to any considerable extent are extremely rare, cause little if any cyanosis, and either give rise to no murmurs or to murmurs quite different in character and propagation from those in this case, we learn from the researches of those pathologists who have studied these malformations that the probability of the original lesion being in the pulmonary artery increases with the age of the sufferer over fifteen. The age of our patient being nineteen, there is therefore a very strong probability that her primary lesion has been in the pulmonary artery, and the marked, rough systolic murmur heard in her pulmonary area, and propagated with most distinctness towards the left axilla, renders this certain. The distinct and accurate closure of the pulmonic valves (absence of diastolic murmur) shows us that the murmur probably depends upon a constriction lying above them; as, if they were so malformed as to obstruct the exit of the blood, they would almost certainly be incompetent also. Now, the certain result of constriction of the pulmonary artery to any extent, or for any considerable period, would inevitably ke a gradually increasing congestion and dilatation of the right ventricle-lengthened period of action being equivalent to increase of constriction; and of the existence of this in the present case we have ample proof in the loud systolic murmur audible over the right apex, and in the distinct jugular pulsation, which shows that the regurgitation through the tricuspid valve has been so persistent and so great that it has destroyed the valvular action of the venous valves at the root of the neck, so as to convert what must at first have been a mere undulatory movement in the venous current into a distinct pulsatory wave. The occurrence of so great a dilatation of the right ventricle sufficiently explains the 
absence of an apex beat, because the dilated right ventricle, lying in front of the left one, acts as a water cushion or buffer in preventing the apex from reaching the thoracic wall; while the absence of distinct pulsation in the scrobulus cordis, and at the lowest part of the sternum, proves that the heart is feeble, and that in the right side dilatation is the prevalent lesion.

Congenital constriction of the pulmonary artery may occur at various periods of intra-uterine life, and the mechanical results on the cardiac development and consequent symptoms must vary accordingly. When it occurs just previous to the completion of the ventricular septum, and to such an extent as to prevent the complete formation of that septum, we have as a natural result the phenomenon of a complete double circulation with free intercommunication between the two ventricles, one of which is connected with an obstructed and the other with a patent arterial conduit. The natural result of this is, that when both ventricles contract with nearly equal force upon nearly equal contents, that one whose natural outflow is obstructed forces a portion of its contents through the abnormally patent channel into the other ventricle whose outlet is unimpeded; and the consequence of this is, forcible dilatation of the unimpeded outlet-in this case the aorta,-and consequently a systolic murmur of tension accompanying the blood wave and followed by a loud accentuated aortic second, the natural result of the forcible closure of the aortic semilunar valves by an unusually heavy column of blood, precisely the usual condition in this case. When, however, from any cause-such as catarrhal congestion-the pulmonary circulation is more than usually obstructed, the result is the same as in the normal condition: the blood-recoil upon the pulmonary valves is greatly increased, and we have an apparent and temporary transference of the accentuation from the aortic to the pulmonic second sound-a phenomenon which repeatedly occurred in this case while the patient remained under observation.

As to the systolic murmur in the mitral area, the absence of distinct propagation round the left side to the back is opposed to the idea of any regurgitation through the mitral valve. The conditions necessary for the production of this regurgitation were, moreover, entirely absent: because free communication between the ventricles prevents the possibility of over-dilatation of the left ventricle and regurgitation from this cause; while congenital mitral constriction, which might give rise to it, would not only be a very unusual complication in such a case, but is also conclueively disproved by the entire absence of any evidence of dilatation of the left auricle, which in all such cases is sure to be revealed by distinct pulsation to the left of the pulmonary area and in the same plane, evincing dilatation and hypertrophy of the left auricular appendix. The systolic murmur over the left apex is therefore due to propagation from the aortic and pulmonary regions, and possibly also to propagation from the opening in the upper part of the ventricular septum, which I have shown to exist.

In this most interesting case, therefore, there exists contraction of the pulmonary artery above the valves, with deficiency of the interventricular septum at its upper part, dilatation of the right ventricle with destruction of the venous valves at the root of the neck, and dilatation of the ascending aorta; and these conditions may be accepted as facts conclusively proved by the phenomena present. But there is one other malformation the presence of which is rendered probable by what we know of the sequential development of the heart, though it is not, and, so far as I know, cannot be, revealed by any diagnostic phenomenaand that is, more or less imperfect occlusion of the foramen ovale. Not only, therefore, has it been shown that in this case the symptoms depend upon congenital malformationan opinion which even a cursory inspection of the patient would lead almost everyone to adopt,- - but it has also been shown that the physical signs present confirm this view, and that these are precisely such as our knowledge of the sequential development of the heart and of the mode in which these physical signs are produced would lead us to expect to be present in a case of pulmonary constriction arising at a certain period of intra-uterine gestation. The ease with which we can now interpret the symptoms and signs in a case so complicated as the present, compared with the difficulties which formerly beset the diagnosis of the simplest valvular lesion, exhibits in a most striking manner the very great advance in the diagnosis of cardiac disease which has taken place during the last thirty years.

I have only to add that, under appropriate treatment, the patient left the infirmary improved in every respect, and that she still remains under observation.

Edinburgh.

\section{ON THE COMPARATIVE ACTION OF ALCOHOL AND ABSINTHE.}

\author{
By Dr. MAGNAN, \\ PHYSICIAT TO THE STR. ANNE ABYLUM, PARIS.
}

Patrents affected with acute alcoholism may offer the following peculiarities :-

1. Some of them present convulsive phenomena, which nothing in the previous symptoms allowed us to foresee. The palient, in fact, all at once loses consciousness, and suddenly falls; the face becomes pale, the limbs stiffen, and the muscles of the trunk and neck contract, so as to turn the head to one side. To this stage of tonic spasm soon succeed clonic spasms, twitchings in the arms and legs, with grimaces of the face, and convulsive movements of the eyes; frothy saliva, occasionally tinged with red, covers the mouth, and the tongue is bitten; then the lips grow blue, the face livid, and the breathing stertorous, whilst the urine and fæces escape involuntarily; a more or less prolonged state of stupor following the fit.

We have here, it is evident, an attack of epilepsy. Such a fit may occur equally at the periods of onset, of full establishment, or of the decline of the attack of delirium tremens-that is to say, in stages of the attack when the trembling is very violent, or, on the other hand, very slight. After the attack the trembling follows its usual course: it diminishes if the attack of delirium tremens is on the decline; it increases, on the contrary, if the fit has come on at the earlier part of the attack.

The fit, then, is not the highest expression of the motor disturbance, nor is it the most advanced degree of that general tremulousness which accompanies delirium tremens; it is an accident of a different order, superadded to the other disturbances of motility, and due to a distinct cause, which from the antecedent history is usually absinthe, more rarely bitters, vermouth, or vin blanc, the adulterated white wine which is sold retail in Paris by most of the wine merchants, and is very different from that drunk in the neighbourhood of Vignobles.

An acquaintance with this fact is not unimportant either from a diagnostic or therapeutic point of view; and perbaps bleeding would have been used more moderately for patients affected with epilepsy wrongly called "alcoholic," if practitioners had been thoroughly impressed with the idea that the attack was due to the direct action of a poison which possesses the special property of stimulating the excitomotor functions of the medulla and spinal cord, without the necessary intervention on the part of the brain of that determination of blood to which certain writers have ascribed the principal rôle in the production of convulsive seizures.

To sum up, the epileptic attack is an exceptional phenomenon due to a special cause, and independent of the degree of trembling.

2. In certain rarer cases, in patients suffering from acute alcoholism, we observe the occurrence of an attack of delirium which presents all the general characters of alcoholism-that is to say, hallucinations of very changeable character, of painful, disagreeable, and aggressive nature, recalling, it may be, either the profession of the individual or the dominant preoccupations of the time of attack. But with this characteristic delirium the trembling in this class of patients is next to nothing; it may even be entirely wanting, so that the attack of acute alcoholism may be found reduced simply to intellectual disturbances. Cases of this kind have been on record for some years past. M. Motet relates some observations of the kind in his thesis of 1859 "On Alcobolism, and the Poisonous Effects produced in Man by the Liqueur Absinthe." In his cases the patients were especially drinkers of absinthe. 